\title{
A critical reappraisal of dietary practices in methylmalonic acidemia raises concerns about the safety of medical foods. Part 2: cobalamin C deficiency
}

\author{
Irini Manoli, MD, PhD'1, Jennifer G. Myles, MS, RD², Jennifer L. Sloan, PhD, MS', \\ Nuria Carrillo-Carrasco, MD ${ }^{1,3}$, Eva Morava, MD, PhD ${ }^{4}$, Kevin A. Strauss, MD ${ }^{5}$, \\ Holmes Morton, $\mathrm{MD}^{5}$ and Charles P. Venditti, MD, PhD ${ }^{1}$
}

\begin{abstract}
Purpose: Cobalamin $\mathrm{C}(\mathrm{cblC})$ deficiency impairs the biosynthesis of $5^{\prime}$-deoxyadenosyl-adenosyl- and methyl-cobalamin, resulting in methylmalonic acidemia combined with hyperhomocysteinemia and hypomethioninemia. However, some patients with $c b l C$ deficiency are treated with medical foods, devoid of methionine and high in leucine content, that are formulated for patients with isolated propionate oxidative defects. We examined the effects of imbalanced branched-chain amino acid intake on growth outcomes in $c b l C$-deficient patients.
\end{abstract}

Methods: Dietary intake was correlated with biochemical, anthropometric, and body composition measurements and other disease parameters in a cohort of 28 patients with early-onset $c b l C$ deficiency.

Results: Protein-restricted diets were followed by $21 \%$ of the patients, whereas $32 \%$ received medical foods. Patients on protein-restricted diets had lower height-for-age $Z$-score $(P=0.034)$, whereas patients consuming medical foods had lower head circumference $Z$-scores $(P=0.037)$, plasma methionine concentrations $(P=0.001)$, and predicted methionine influx through the blood-brain barrier $Z$-score $(-1.29$ vs. $-0.0617 ; P=0.007)$. The combination of age at diagnosis, a history of seizures, and the leucine-to-valine dietary intake ratio best predicted head circumference $Z$-score based on multiple regression modeling $\left(R^{2}=0.945\right)$.

Conclusions: Patients with $c b l C$ deficiency treated with medical foods designed for isolated methylmalonic acidemia are at risk for iatrogenic methionine deficiency that could adversely affect brain growth and development.

Genet Med advance online publication 13 August 2015

Key Words: cobalamin C (cblC) deficiency; dietary guidelines; medical foods; methionine; methylmalonic acidemia

\section{INTRODUCTION}

Combined methylmalonic acidemia and hyperhomocysteinemia, cobalamin $\mathrm{C}(\mathrm{cblC})$ type, results from impaired biosynthesis of 5'-deoxyadenosyl- and methyl-cobalamin, cofactors for the methylmalonyl-CoA mutase and methionine synthase enzymes, respectively. ${ }^{1-3}$ Although the biochemical phenotype of this disorder shares elevated methylmalonic acid concentrations with the classic mut subtype of isolated methylmalonic acidemia (MMA), the propionate-derived metabolic perturbations are of lesser magnitude, whereas the clinical findings and disease pathophysiology are distinct. CblC-deficient patients display significantly increased homocysteine and decreased methionine concentrations, and patients with early onset present with failure to thrive, visual impairment due to macular degeneration/pigmentary retinopathy, hemolytic uremic syndrome and thromboembolic/microangiopathic complications, cardiopulmonary findings, neurocognitive delay, seizures, and other neurological manifestations. ${ }^{4-8}$ Decreased methionine synthesis and secondary impairments of the remethylation cycle, methyltransfer reactions, homocysteine-thiolactone metabolism, and increased oxidative stress have been implicated in disease pathophysiology., ${ }^{5,9-11}$ The mechanisms underlying the multisystemic manifestations of $c b l C$ deficiency remain unknown, in part because of the lack of viable animal models. ${ }^{12}$

Treatment remains particularly challenging in $c b l C$-deficient patients because symptoms such as intrauterine growth retardation, microcephaly, mild craniofacial dysmorphism, and congenital heart disease can develop in utero. ${ }^{13-16}$ Moreover, despite early diagnosis by newborn screening, clinical manifestations, including the characteristic ocular and neurological complications, are at best incompletely responsive to conventional therapies. ${ }^{4,6,7,9}$ The mainstay of treatment involves high-dose intramuscular hydroxocobalamin injections that can ameliorate the biochemical perturbations, resulting in a decrease of homocysteine and methylmalonic acid concentrations and an increase in methionine synthesis. Betaine supplementation also enhances remethylation of homocysteine to methionine via the betaine:homocysteine methyltransferase reaction. Folinic

\footnotetext{
'Organic Acid Research Section, Genetics and Molecular Biology Branch, National Human Genome Research Institute, National Institutes of Health, Bethesda, Maryland, USA; ${ }^{2}$ Nutrition Department, Clinical Center, National Institutes of Health, Bethesda, Maryland, USA; ${ }^{3}$ Therapeutics for Rare and Neglected Diseases, National Center for Advancing Translational Sciences, National Institutes of Health, Bethesda, Maryland, USA; ${ }^{4}$ Tulane University Medical School, New Orleans, Louisiana, USA; ${ }^{5}$ Clinic for Special Children, Strasburg, Pennsylvania, USA. Correspondence: Charles P. Venditti (venditti@mail.nih.gov)

Submitted 3 March 2015; accepted 22 June 2015; advance online publication 13 August 2015. doi:10.1038/gim.2015.107
} 
or folic acid and other supplements (carnitine, methylcobalamin, pyridoxine, methionine) have been used, yet longitudinal efficacy studies of disease-related clinical outcomes remain uncertain. ${ }^{9}$

Protein restriction can be considered to decrease the propiogenic amino acid load (valine, isoleucine, threonine, and methionine) and help lower methylmalonic acid production. ${ }^{17}$ However, methylmalonic acid concentrations in $c b l C$-deficient patients are typically orders of magnitude less than those seen in isolated patients with mut MMA, consistent with the observations that metabolic decompensations of the "intoxication" type seen in classic isolated MMA are very rare in patients with $c b l C$ deficiency. ${ }^{18,19}$ Furthermore, protein restriction carries the risk of inducing iatrogenic methionine, and methyl-donor ( $S$-adenosylmethionine) deficiency, resulting in the impairment of multiple biological transmethylation reactions such as creatine, phosphatidylcholine, and sphingomyelin biosynthesis, as well as DNA methylation., ${ }^{920-22}$ This could adversely affect growth and neurodevelopmental outcomes, as evidenced by the severe neurological phenotypes variably seen in patients with other remethylation disorders, including $5^{\prime}, 10^{\prime}$-methylenetetrahydrofolate reductase deficiency, functional methionine synthase (cblE/G) deficiencies, or other intracellular cobalamin biosynthesis disorders $(c b l D, F, J))^{1,21,23,24}$

Over the course of several years of evaluating $c b l C$-deficient patients via a natural history protocol, we observed a highly variable approach to the dietary management of $c b l C$ deficiency. Dietary practices ranged from following a completely unrestricted diet to prescribing strict protein restriction, supplemented by large quantities of medical foods. The surprisingly large number of $c b l C$-deficient patients who are managed with medical foods designed for isolated MMA and propionic acidemia (PA) in our study and the published literature ${ }^{6}$ led us to critically reappraise the dietary management of $c b l C$ deficiency. We find that the use of medical foods in $c b l C$-deficient patients is associated with iatrogenic methionine and essential branched-chain amino acid deficiencies, impaired growth, and abnormal body composition.

\section{METHODS}

\section{Study population}

Patient studies were approved by the National Human Genome Research Institute Institutional Review Board and performed in compliance with the Helsinki Declaration (ClinicalTrials. govNCT00078078). Patients were enrolled between 2004 and 2014 from centers across the United States and Canada. Long-term dietary management was dictated by each patient's metabolic center. Study participants had no clinical symptoms or laboratory markers of metabolic instability during their National Institutes of Health evaluations. The diagnosis of $c b l C$ deficiency was confirmed using cellular biochemistry (in the laboratory of Dr. David S. Rosenblatt, Division of Medical Genetics, McGill University, Montreal, Canada) and/ or through molecular genetic analysis of the $M M A C H C$ gene (GeneDx, Gaithersburg, MD). ${ }^{25}$

\section{Laboratory studies}

The National Institutes of Health Clinical Center laboratory performed routine laboratory investigations, including total homocysteine and vitamin B12 concentrations. Total homocysteine was determined by high-performance liquid chromatography between 2004 and 2013 and by an enzymatic assay (Cobas 6000 analyzer; Roche Diagnostics Indianapolis, IN) from 2013 to present. Plasma and urine methylmalonic acid and amino acid concentrations were determined by liquid chromatography-tandem mass spectrometry (Mayo Medical Laboratories Rochester, MN). Samples were obtained in the fasting state, or 2-4 hours after a meal, whenever possible.

\section{Dietary regimens and daily amino acid intake calculations}

Dietary analysis was performed using the patient's medical foods (specialized formula) prescriptions and a detailed diet history obtained by a research dietitian. A 3-day food record completed by the families was available for 16 of 28 patients.

Dietary analyses and calculations were performed using Nutrition Data System for Research software versions 2007-2012 developed by the Nutrition Coordinating Center (University of Minnesota, Minneapolis, MN). ${ }^{26}$ Medical food composition information was obtained from the respective manufacturers. Recommended dietary allowance (RDA) for protein and amino acids were based on the dietary reference intakes for protein and amino acids. ${ }^{27}$ Protein intake was recorded as complete protein from natural protein sources and incomplete/deficient protein-equivalent intake (grams/ kilogram/day) in the form of specialized MMA/PA medical foods, such as Propimex1/2 (Abbott Laboratories, Columbus, $\mathrm{OH}$ ); XMTVI Analog; Maxamaid or Maxamum (Nutricia North America, Cedar Knolls, NJ); and MMA/PA Express (Vitaflo USA, Alexandria, VA). Amounts of individual propiogenic amino acids (valine, isoleucine, methionine, and threonine), as well as leucine, were recorded for patients with detailed dietary records $(n=13)$, whereas estimated deficient versus complete protein intake (grams/kilogram/day) was documented for all patients.

Patients received intramuscular hydroxocobalamin therapy with different therapeutic dosages, ranging between 0.01 and $0.61 \mathrm{mg} / \mathrm{kg} / \mathrm{day}$. All patients received betaine at dosages ranging from 31 to $338 \mathrm{mg} / \mathrm{kg} / \mathrm{day}$, as well as folate or folinic acid supplementation, whereas a minority were prescribed aspirin, fish oil, docosahexaenoic acid (DHA), or other dietary supplements. These data are provided for each patient, along with additional nutritional information, in Supplementary Table S1 online.

\section{Predicted cerebral amino acid influx calculations}

Cerebral influx of substrates for the cationic $\left(\mathrm{y}^{+}\right.$; lysine, arginine, ornithine) and large neutral amino acid transport systems (LAT1; glutamine, histidine, isoleucine, leucine, methionine, phenylalanine, threonine, tryptophan, tyrosine, and valine) was estimated as previously described for patient cohorts with $5^{\prime}, 10^{\prime}$-methylenetetrahydrofolate reductase deficiency, maple 
syrup urine disease, and glutaric acidemia. ${ }^{21,28,29}$ These calculations were based on the principle that the cerebral uptake of each amino acid is influenced by the ambient (plasma) concentrations of its competitors. Substrate competition is expressed by an apparent $K_{\mathrm{m}}$, called $K_{\text {app }}(\mu \mathrm{mol} / \mathrm{l})$, calculated for each amino acid according to the equation: $K_{\text {app }}=K_{\mathrm{m}}\left[1+_{\Sigma 1 \rightarrow \mathrm{n}}(\mathrm{Ci} / \mathrm{Ki})_{n}\right] . K_{\mathrm{m}}$ is the classical Michaelis-Menten affinity constant for the single amino acid of interest, $C_{\mathrm{i}}$ is the plasma concentration (micromoles/ll) for each of $n$ competitors, and $K_{\mathrm{i}}$ is the classical affinity constant of that competitor (micromoles/l)..$^{30}$ The $K_{\text {app }}$ value then was used to calculate the brain influx (nanomoles/minute/gram of brain tissue) of each amino acid in the competing group, according to the equation: Influx $=\left(V_{\max }\right)(C) /\left(K_{\text {app }}+C\right)$, where $V_{\max }$ and $C$ are the maximal transport velocity (nanomoles/gram/ minute) and plasma concentration (micromoles/l), respectively, of each amino acid. Estimated brain influx $Z$-score values were calculated using mean values observed in a control population of 52 children not affected by disorders of amino acid, folic acid, or cobalamin metabolism from the Clinic for Special Children (Strasburg, PA).

\section{Anthropometry, body composition, and neurocognitive evaluations}

Anthropometric measurements were expressed as age- and sexspecific $Z$-scores using the epidemiological software package Epi Info (http://wwwn.cdc.gov/epiinfo/), based on the Centers for Disease Control and Prevention 2002 reference database (Atlanta, GA) for patient under 20 years of age. Whole-body composition in grams of fat or fat mass, and fat-free (or lean) body mass, were measured using dual-energy X-ray absorptiometry (Delphi A; Hologic, Bedford, MA). For patients between 5 and 23 years old, a height-adjusted $Z$-score was calculated using the Bone Mineral Density in Childhood Study online calculator (http://www.bmdcspublic.com/zscore.htm).

\section{Data analysis}

The results are presented as means $( \pm \mathrm{SD})$. Significance was set at $P<0.05$. Statistical manipulations were performed using SPSS version 21.0 (IBM, Chicago, IL) or GraphPad Prism software version 6.0 (GraphPad Software Inc., San Diego, CA). An independent Student $t$-test was used to compare values between patients who had or had not been receiving medical foods. Pearson correlation coefficient and multiple linear regression analyses were used to evaluate correlations between variables. Dummy variables were generated for dichotomous categorical variables such as sex, seizures, or congenital microcephaly to be used in the regression model.

\section{RESULTS}

\section{Anthropometric characteristics and body composition}

We studied 28 patients with early-onset $c b l C$ deficiency (18 male, 10 female; age range: $2.03-27.04$ years; mean age \pm SD: $10.2 \pm 7.61$ years). Age at diagnosis varied widely, from the first week of life $(n=4)$ to 24 months (mean \pm SD: $2.62 \pm 4.4$ months; median: 1.5 months). Seventeen of 28 patients $(60 \%)$ were homozygous for the common c.271dupA, p.R91KfsX14 mutation in the MMACHC gene, which is associated with a severe early-onset phenotype (Table 1). Height, weight, body mass index, and head circumference (occipitofrontal circumference $(\mathrm{OFC})$ )-for-age $Z$-scores (mean $\pm \mathrm{SD}$ ) are presented (Table 1 ). Mean $Z$-score for height was $-1.04 \pm 1.33$ (range: 1.8 to -3.5 ); weight, $-0.66 \pm 1.83$ (range 3.00 to -6.8 ); body mass index, $0.13 \pm 1.47$ (range: 2.6 to -4.4 ); and OFC, $-1.57 \pm 1.23$ (range: 0.32 to -4.40 ; median: -1.06 ). Height and OFC $Z$-scores less than -2.00 were found in six and seven patients, respectively. Of note, three of the patients had a history of congenital microcephaly, whereas 9/28 (32.14\%) had a history of seizures, 2 of whom had a diagnosis of infantile spasms (Table 1). Growth outcomes were not different between male and female subjects, or between patients homozygous for the c.271dupA mutation versus others.

Whole-body composition analysis based on dual-energy $\mathrm{X}$-ray absorptiometry imaging studies was available for 18 patients (12 males and 6 females). Mean \pm SD percentage of fat mass was $31.56 \pm 11.2 \%$, with $27.9 \pm 8.86 \%$ in males and $38.11 \pm 12.85 \%$ in females $(P=0.07)$. Mean bone mineral density height-adjusted $Z$-score was $-1.9 \pm 1.55$, with $-1.67 \pm 0.93$ for males and $-2.46 \pm 2.77$ for females ( $P=$ not significant).

\section{Correlations of growth outcomes with protein restriction and medical foods utilization}

Six of $28(21 \%)$ of the patients received complete protein below $85 \%$ of the RDA for healthy children. ${ }^{27}$ Nine of the 28 patients (32.14\%) received medical foods designed for MMA. Additional protein-free $(n=5)$ or elemental amino acid formulas $(n=2)$ were used in a minority of patients, whereas one patient required valine supplementation for persistently low valine plasma concentrations (Table 1 ).

Comparing growth and biochemical parameters between patients following a protein-restricted diet or receiving medical foods versus those who were not, it became evident that protein restriction or chronic medical food use were associated with poorer growth parameters. Patients following a diet with less than $85 \%$ the RDA for complete protein had lower height-for-age $(-2.16 \pm 1.04$ vs. $-0.72 \pm 1.24 ; n=5$ vs. 17 ; $P=0.030)$ and head circumference (OFC)-for-age $Z$-scores $(-2.3 \pm 1.30$ vs. $-1.2 \pm 1.11 ; P=0.062)$ and a higher percentage of fat mass (and hence lower lean or skeletal muscle mass) by dual-energy X-ray absorptiometry analysis $(43.1 \pm 10.8$ vs. $26.7 \pm 7.3 \% ; n=12$ and $5 ; P=0.002$ ). Similarly, a lower mean weight-for-age $(-1.7 \pm 2.28$ vs. $-0.09 \pm 1.3 ; P=0.038$; $n=15$ and 8 , respectively), height-for-age $(-1.72 \pm 1.01$ vs. $-0.73 \pm 1.37 ; P=0.075)$, and head circumference-for-age $Z$-score $(-2.48 \pm 1.43$ vs. $-1.15 \pm 0.88 ; P=0.037)$ was observed in the subset of patients consuming medical foods (Figure 1a). Patients with a history of seizures had a significantly lower height and OFC $Z$-score than those without seizure history $(P=0.004$ and $<0.001$, respectively) (Figure $1 b)$. Growth outcomes (height or OFC $Z$-scores) in patients who carried no, one, or two copies of the common c.271dupA mutation were 


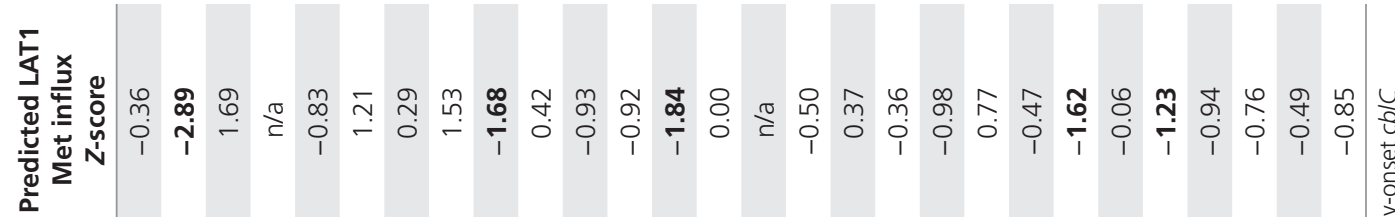

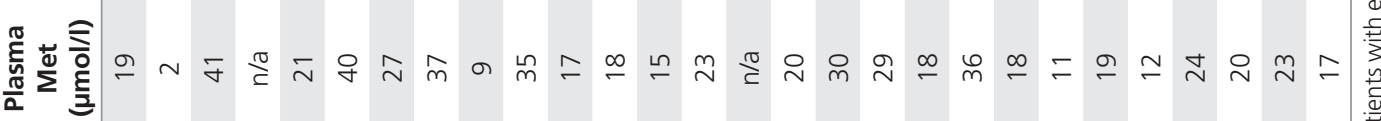

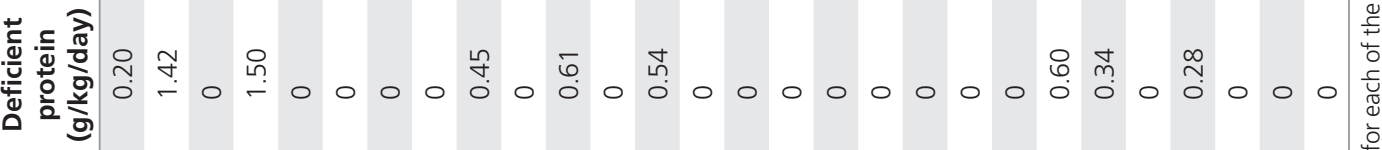

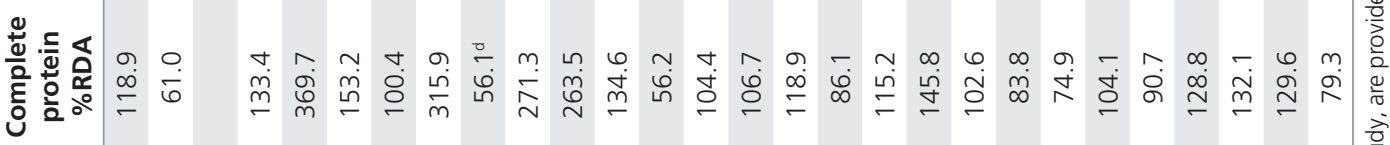

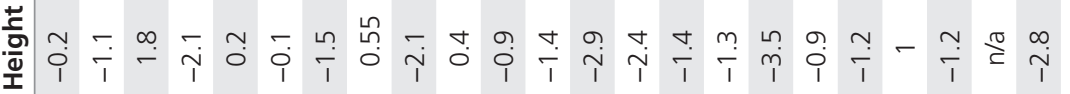

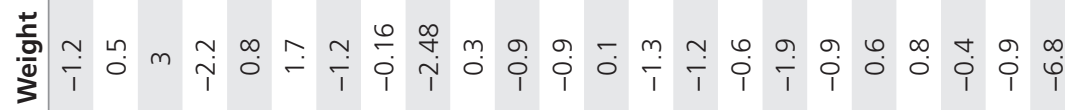

$\frac{8}{8}$

兵

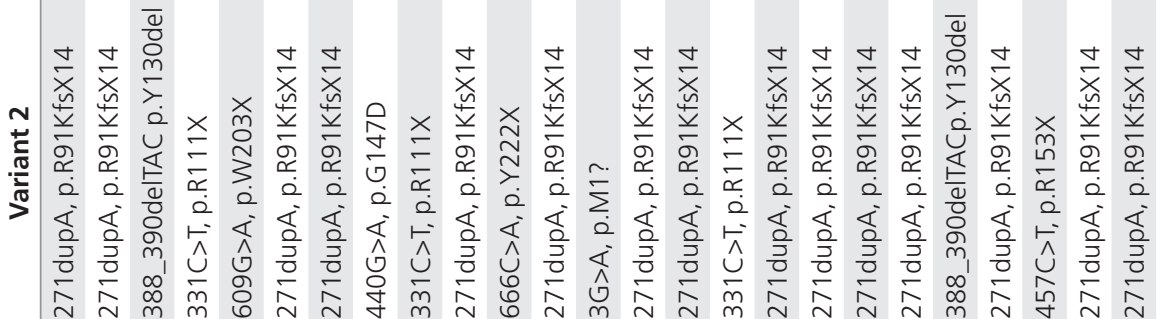




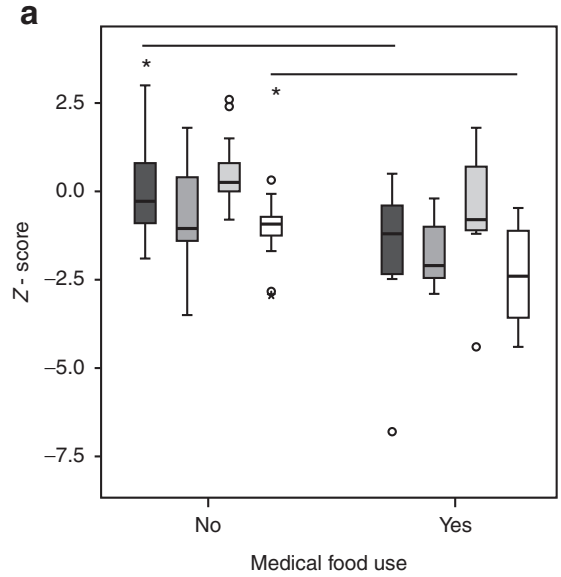

$\square$ Weight $\square$ Height $\square$ BMI $\square$ OFC

d

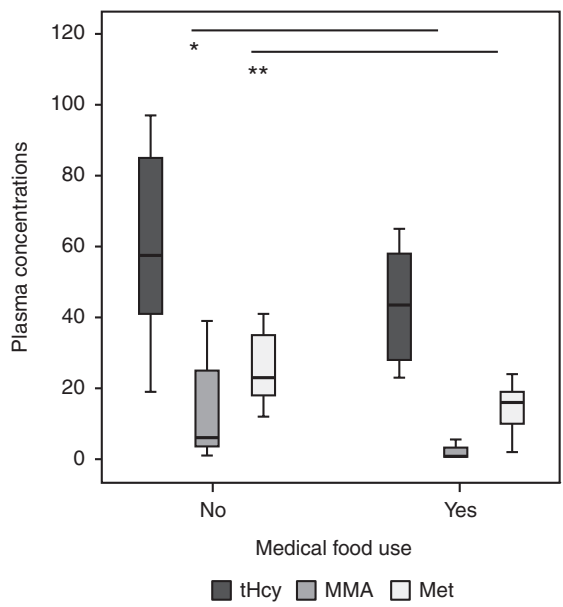

b

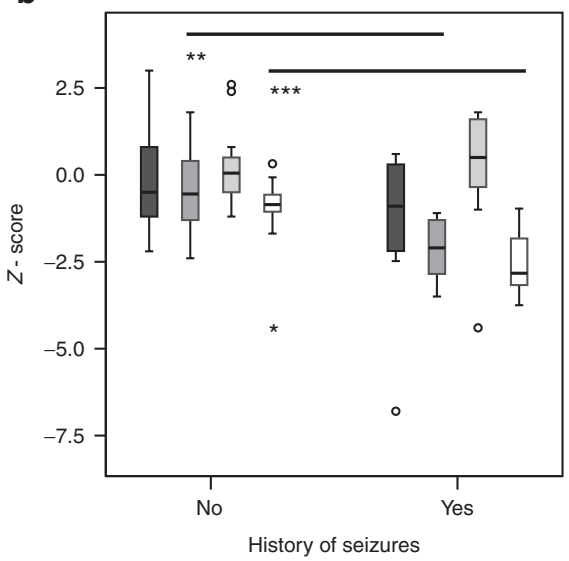

$\square$ Weight $\square$ Height $\square$ BMI $\square$ OFC

e

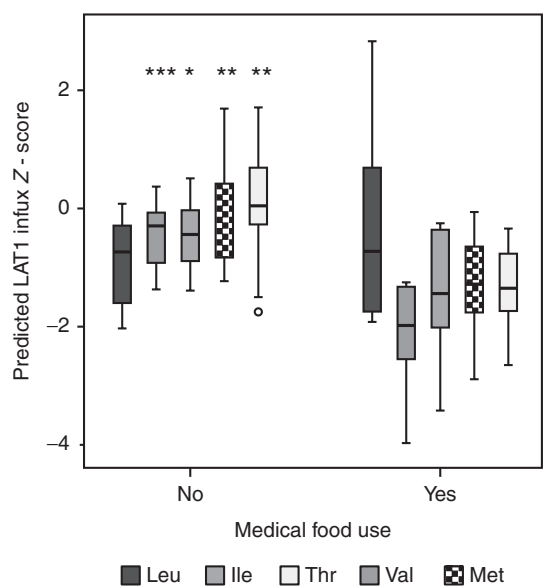

C

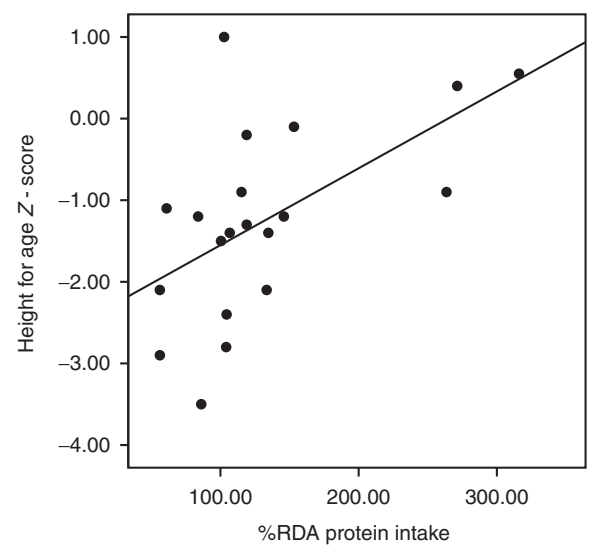

f

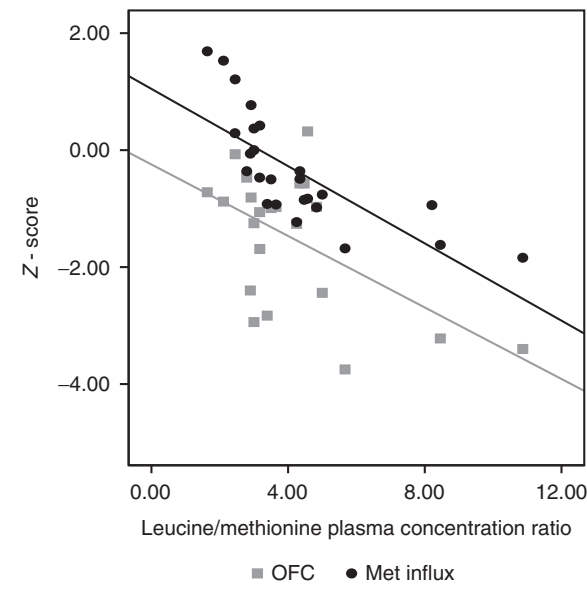

Figure 1 Growth parameters, serum metabolites and predicted amino acid influx through the blood brain barrier. Association with medical food vs. complete protein intake, history of seizures. (a) A lower weight-for-age $(-1.7 \pm 2.28$ vs. $-0.09 \pm 1.3 ; P=0.038 ; n=15$ and 8 , respectively) and head circumference-for-age $Z$-score $(-2.48 \pm 1.43$ vs. $-1.15 \pm 0.88 ; P=0.037)$ and a trend towards a lower mean height-for-age $(-1.72 \pm 1.01$ vs. $-0.73 \pm 1.37$; $P=0.075)$ and body mass index-for-age $Z$-score $(-0.60 \pm 2.08$ vs. $0.48 \pm 0.99 ; P=$ not significant $)$ was observed in the group consuming medical foods compared with patients who did not at the time of evaluation. (b) Patients with a history of seizures had a significantly lower height and OFC Z-scores than those without seizure history ( $P=0.004$ and $<0.001$, respectively). (c) Complete protein intake as a percentage of the recommended daily allowance (RDA) correlated positively with height-for-age $Z$-score $\left(r=0.575 ; P=0.008 ; R^{2}=0.31\right)$. (d) Patients consuming medical foods had slightly lower total homocysteine concentrations ( $48.7 \pm 27.7$ vs. $59.8 \pm 25.2 ; P=$ not significant), significantly lower plasma methylmalonic acid $(2.75 \pm 3.27$ vs. $13.9 \pm 16.4 ; n=9$ vs. 18 ; $P=0.012)$ and methionine concentrations ( $14.5 \pm 6.92$ vs. $25.8 \pm 8.8 ; P=0.004)$, as expected; and lower valine and isoleucine concentrations $(P<0.001$ and 0.026 , respectively; data not shown), compared with patients not consuming medical foods. (e) Predicted influx through the blood-brain barrier transporter LAT1 for the propiogenic amino acids and leucine is provided as a Z-score from mean values derived from plasma amino acid concentrations in a control population of 52 children from the Clinic for Special Children. Patients consuming medical foods had mean predicted influx Z-scores lower than those not consuming special formulas (valine: $-2.11 \pm 0.92$ vs. $-0.47 \pm 0.56 ; P=0.001$; isoleucine: $-1.41 \pm 1.09$ vs. $-0.45 \pm 0.56 ; P=0.045$; methionine: $-1.29 \pm 0.90$ vs. $-0.61 \pm 0.89 ; P=0.004$; and threonine: $-1.33 \pm 0.72$ vs. $0.06 \pm 0.87 ; P=0.001)$. (f) The leucine-to-methionine plasma concentration ratio correlated negatively with the predicted cerebral methionine influx $\left(r=-0.749 ; R^{2}=0.541 ; P<0.001\right)$ as well as the head circumference-for-age $Z$-scores $\left(r=-0.553 ; R^{2}=0.306\right.$; $P=0.008 * P<0.05, * * P<0.01, * * * P<0.001)$.

not significantly different $(P=0.876$ and 0.591 , respectively, one-way analysis of variance), suggesting nongenic effects have a significant contribution to the variance observed. Similarly, no significant difference was observed in the percentage RDA of natural protein intake or the use of medical foods (grams/ kilograms/day) between patients with early-onset $c b l C$ deficiency who have different genotypes.

Complete protein intake (percentage RDA) showed a positive correlation with the height-for-age $Z$-score
(Figure 1c). Given that MMA/PA medical foods contain no propiogenic amino acid, but a normal to increased amount of leucine, ratios of leucine to methionine or valine dietary intake were significantly elevated in patients consuming medical foods ( $P=0.015$ and 0.003 , respectively). A higher leucine-to-valine dietary intake showed a negative correlation to the height-for-age $(r=-0.673 ; P=0.033$; $\left.R^{2}=0.453\right)$ and OFC-for-age $Z$-scores $(r=-0.840 ; P=0.001$; $\left.R^{2}=0.705\right)$. 
Intakes of other nutrients that can affect growth and cognitive function, including iron, zinc, and omega 3 fatty acids, were also assessed (Supplementary Table S1 online). All subjects well surpassed the RDA for iron from dietary intake plus supplements. Three subjects had intake of zinc below the RDA, but none of these subjects had growth failure. Nine subjects had alpha-linolenic acid below the adequate intake. Two of the nine were taking DHA or fish oil supplements to compensate; the remaining seven had negligible DHA intake.

\section{Biochemical indexes and cerebral amino acid influx calculations}

Since protein restriction and medical food supplementation are both used to improve metabolic control, we looked at the effects of the dietary management on plasma metabolite concentrations. Patients who received medical foods had significantly lower plasma methylmalonic acid and methionine concentrations (Figure 1d), as well as lower valine and isoleucine (data not shown). Total homocysteine was not significantly different. The higher ratio of leucine over methionine, valine, and isoleucine dietary intake also resulted, predictably, in elevated leucine/methionine $(P=0.05)$ and leucine/valine $(P=0.002)$ plasma amino acid concentrations (similarly, elevated ratios of tyrosine or phenylalanine to any of the restricted amino acids were observed).

These skewed plasma amino acid ratios are predicted to alter amino acid transport across cells and physiological barriers through competitive inhibition at shared transporters/transport systems, such as the leucine-preferring large neutral amino acid transporter type 1, LAT1 (SLC7A5). To investigate whether these skewed ratios would affect the influx of amino acids at the blood-brain barrier (BBB) via the LAT1 transporter, we applied the cerebral influx model using plasma amino acid concentrations from our $c b l C$-deficient patient cohort. ${ }^{30}$ We document $Z$-scores for methionine influx ranging from 1.69 to -2.89 , with $18 / 28(64 \%)$ having a negative $Z$-score, and 5/27 (18\%) having a score $<-1.00$ (Table 1$)$. The range of values for valine ( 0.37 to -3.97 ), isoleucine ( 0.51 to -3.42$)$, and threonine ( 1.71 to -2.65 ) are also reported. These $Z$-scores were significantly lower in patients receiving medical foods compared with those who did not (Figure 1e), whereas methionine (and valine) BBB influx $Z$-scores was significantly lower among patients with a history of seizures (for methionine: -0.035 for no seizure history $(n=16)$ vs. -1.087 in patients with seizures $(n=10) ; P=0.012)$. The leucine-to-methionine plasma concentration ratio correlated negatively with the predicted cerebral methionine influx $(P<0.001)$ as well as the head circumference-for-age $Z$-scores $(P=0.008)$ (Figure 1f).

We present an illustrative case study of an infant, who presented with severe encephalopathy and hyperammonemia and was therefore treated with protein restriction and MMA/PA medical foods, along with daily intramuscular hydroxocobalamin and adequate doses of betaine and carnitine. The infant showed a decline in head size and length centiles, from the 33rd to the 2 nd centile for the head circumference and from the 60th to the 8th for length, with no significant improvement in growth until the age of 5 months. This trend was reversed upon increasing the relative ratio of natural to deficient protein intake and changing briefly to a maple syrup urine disease formula (containing minimal amounts of branched-chain amino acids but normal concentrations of methionine) (Supplementary Figure S1 online).

Stepwise multiple regression modeling showed that the combination of age at diagnosis ( $\beta$ coefficient $=-0.295 ; P=0.031$ ), history of seizures $(\beta$ coefficient $=-0.324 ; P=0.034)$, and leucine-to-valine intake ratio $(\beta$ coefficient $=-0.754 ; P<0.001$ ) best predicted OFC $Z$-score, with a model $R^{2}$ of 0.944 . The addition of the dietary component (leucine-to-valine intake ratio) improves the $R^{2}$ of the model from 0.4 to 0.944 , suggesting that diet contributes significantly to the variance observed in the OFC Z-score.

\section{DISCUSSION}

This study detailed the growth outcomes and dietary management of a large cohort of $c b l C$-deficient patients evaluated as part of a dedicated, single-center, natural history protocol (ClinicalTrials.gov NCT00078078). The ability to enroll patients treated at a large number of metabolic clinics in North America afforded a broad representation of the varied therapeutic practices used and the outcomes observed, and provided a large data set of current management approaches.

Protein restriction in $c b l C$ deficiency is intended to lower the amino acid load into the propionate oxidation pathway that is partially blocked at the methylmalonyl-CoA mutase (MUT) step as a result of impaired biosynthesis of the cofactor for MUT, 5'-deoxyadenosylcobalamin. Providing an appropriate dose of parenteral hydroxocobalamin usually leads to a significant decrease in plasma methylmalonic acid concentrations, which are generally 10 - to 100 -fold less than those observed in patients with mut deficiency. Despite the minimal effects on influencing metabolites in the face of hydroxocobalamin therapy, additional protein restriction and administration of propiogenic amino acid-free medical foods, containing minimal amounts of valine, isoleucine, threonine, and, importantly, methionine, were frequently used to treat the patients. In this study we documented that a number of $c b l C$-deficient patients follow a diet restricted in protein $(21 \%)$ and/or supplemented with medical foods designed for MMA/PA (32\%; Table 1). The use of MMA/PA medical foods, although not universally adopted, seems to have gained support in some metabolic centers, as evidenced by the fact that $76 \%$ of $c b l C$-deficient patients identified by newborn screening were prescribed medical foods in one study. ${ }^{6}$ Our data support the concept that protein restriction (and/or medical foods use) to lower the plasma methylmalonic acid concentration has little effect on total homocysteine concentration, but, importantly, it can lower methionine and essential branched-chain amino acid concentrations.

Hypomethioninemia is considered a critical component of $c b l C$ disease pathophysiology, and maximizing methionine synthesis, and the downstream provision of methyl groups, is 
the aim of the two major therapeutic modalities for the disorder, specifically high-dose vitamin B12 (hydroxocobalamin) and betaine. Brain white matter demyelination and subacute combined degeneration have been observed in several case studies/series of $c b l C$ deficiency, ${ }^{6,9,24,31,32}$ resembling findings in animal models with B12 deficiency. The animals' symptoms and pathology could be mitigated by methionine supplementation. ${ }^{33}$ Moreover, methionine supplementation was claimed to improve photoreceptor sensitivity (on electroretinography) and retinal function in a single patient, but it had no effect on the characteristic macular degeneration of the disease. ${ }^{34}$ The pathophysiology of $c b l C$ deficiency is certainly complex because it cannot be reversed or prevented simply by methionine supplementation or any currently available therapies. ${ }^{57,9,23}$ Furthermore, decreased transmethylation products, such as choline, creatine, or guanidinoacetate, have not been consistently aberrant in magnetic resonance spectroscopy or serum/ urine biochemical studies in $c b l C$-deficient patients, ${ }^{6,35}$ and a paradoxical increase in $S$-adenosylmethionine has been noted, postulated to result from secondary inhibition of methylases through adenosylhomocysteine or other metabolites. ${ }^{22,35}$

Despite the unresolved underlying pathophysiology of $c b l C$ deficiency, and the lack of evidence in favor of additional methionine supplementation, a basic therapeutic goal remains to avoid essential amino acid deficiencies, including methionine, especially during periods of brain development. Brain methionine concentrations depend on uptake through the circulation, as well as from de novo methionine synthesis. ${ }^{36}$ Given that MMA/PA medical foods contain no methionine, they may exacerbate methionine deficiency, given the already impaired de novo methionine synthesis that is a feature of $c b l C$ deficiency. Therefore, there is no theoretical rationale or experimental support for the practice of administering medical foods to patients with $c b l C$ deficiency or any disorder that impairs the remethylation of homocysteine. Furthermore, MMA/PA medical foods contain normal to increased amounts of leucine, which, when administered out of proportion to the other branched-chain amino acids, carries the risk of inducing iatrogenic amino acid deficiencies, probably as a result of alpha ketoisocaproic acid (KIC)-induced branched-chain ketoacid dehydrogenase kinase inhibition and increased valine and isoleucine oxidation rates. ${ }^{37}$ Moreover, branched-chain amino acid imbalances exert a multitude of effects in different organ systems, as detailed in our related article ("A critical reappraisal of dietary practices in methylmalonic acidemia raises concerns about the safety of medical foods-Part 1: isolated methylmalonic acidemias"), and summarized in extensive reviews of branched-chain amino acid metabolism. ${ }^{28,37,38}$ The complexity of leucine effects and the tight coupling with isoleucine and valine metabolism have been overlooked in the design of MMA/PA medical foods, perhaps because leucine does not generate propionyl-CoA and is therefore considered "safe" in patients with MMA and PA. In the case of $c b l C$ deficiency, however, the imbalanced intake of leucine and other large neutral amino acids relative to methionine is predicted to result in competitive inhibition for the uptake of dietary methionine at the $\mathrm{BBB}$, and further accentuate methionine deficiency in the brain/central nervous system of patients. Using the plasma amino acid concentrations observed in our cohort of $c b l C$ deficient patients as the data set for the predictive equations used to study maple syrup urine disease, we indeed document a lower calculated influx for methionine into the central nervous system among patients on medical foods compared with those not consuming special formulas (Figure 1e). Moreover, the leucine-to-methionine ratio correlated negatively with the head circumference $Z$-scores (Figure 1f). While the cerebral amino acid influx model takes into account only two of the multiple transport systems at different physiological barriers, such as the gastrointestinal system and $\mathrm{BBB}$, the principle has been used with success in other disorders. ${ }^{21,28,29}$ Manipulations of brain amino acid influx have gained experimental support in animal studies of glutaric aciduria type 1, where lysine uptake in the brain through the $\mathrm{y}^{+}$transport system (SLC7A1-3) was modified with homoarginine or arginine supplementation, leading to lower lysine concentrations in brain homogenates, as well as improved disease-related outcomes. ${ }^{39,40}$ Moreover, modulation of brain methionine and $S$-adenosylmethionine concentrations with dietary methionine modifications has been demonstrated in animal models, ${ }^{36}$ whereas increased methionine synthesis mediated via betaine supplementation was shown to increase methionine and $S$-adenosylmethionine concentrations in the cerebrospinal fluid, reduce cellular Hcythiolactone formation, and improve brain growth in a cohort of patients with $5^{\prime}, 10^{\prime}$-methylenetetrahydrofolate reductase deficiency. ${ }^{21}$ Although LAT1 and $\mathrm{y}^{+}$are discussed here from a proof-of-concept perspective, there are multiple additional transport systems that control amino acid flux between the gastrointestinal system, kidney, systemic circulation, and brain compartment. For example, B ${ }^{0} \mathrm{AT} 1$ (SLC 6A19) and $\mathrm{ATB}^{0+}$ (SLC6A14) are transporters of large aliphatic amino acids, including leucine, valine, isoleucine, and methionine, in the kidney, gastrointestinal tract, and the $\mathrm{BBB}$, respectively. ${ }^{41-43}$ The complex interactions among these various systems and how metabolic foods deficient in selected amino acids may interfere with amino acid flux between different organs remains largely unexplored.

A delay in symptomatic diagnosis, a history of seizures, and a high relative leucine-to-valine dietary intake were the main predictors of a lower head circumference $Z$-score in our multiple regression modeling. Although assigning a cause-and-effect relationship between the different variables is not possible with the cross-sectional approach used here, the strong correlation observed between dietary parameters and plasma methionine concentrations, together with the considerations regarding the leucine competition for transport of methionine across the BBB, suggests that these medical foods could contribute to a poor neurocognitive outcome and provide a strong theoretical argument against the use of medical foods with high leucine and absent methionine in patients with $c b l C$ deficiency. If protein restriction is deemed necessary, for example, at presentation or 
during a rare metabolic crisis with hyperammonemia, medical foods designed for maple syrup urine disease-e.g., Ketonex (Abbott Nutrition) and MSUD Analog (Nutricia) - that contain no branched-chain amino acids (no leucine, valine, or isoleucine) but have a regular amount of methionine are a safer choice for managing patients with cblC deficiency. Further studies need to be designed to estimate the effects of methionine or other methyl-donor supplementation on disease outcome.

In addition to the protein restriction considerations outlined in detail here, other aspects of the dietary needs in this population remain largely understudied, such as essential fatty-acid intake. Alpha-linolenic acid and DHA intake were low to negligible in $9 / 16$ (56\%) of the patients for whom sufficient data were available. Erythrocyte phospholipid and plasma content of DHA and amino acids may be lower in inborn errors of metabolism secondary to reduced intake on restricted diets ${ }^{44}$ and/or interference with conversion of alpha-linolenic acid to DHA and amino acids secondary to toxic metabolites. ${ }^{45}$ Six of seven subjects with inadequate alpha-linolenic acid and DHA intake followed a restricted diet related to prescribed protein restriction or had poor intake for other reasons. It is well recognized that omega-3 fatty acids are important for optimal cognitive function. Furthermore, omega-3-fatty-acid deficiency can also affect platelet aggregation, an important consideration in patients with hyperhomocysteinemia who are prone to thromboembolic complications. ${ }^{46,47}$ Monitoring of dietary intake and plasma concentrations should be incorporated in the management of $c b l C$-deficient patients.

Our study emphasizes the need to develop disease-specific guidelines under the American College for Medical Genetics and Genomics ACT sheet and algorithm for the follow-up of increased propionylcarnitine (C3) detected by newborn screening to more clearly distinguish isolated MMA from early cobalamin biosynthetic defects, because the dietary management should be distinct. In addition, the studies presented here highlight the paucity of experimental and clinical trial support underlying dietary management practices commonly used to treat patients with varied forms of MMA and underscore the community need for a more rigorous scientific and clinical study of medical foods in the treatment of patients with inborn errors of metabolism.

\section{SUPPLEMENTARY MATERIAL}

Supplementary material is linked to the online version of the paper at http://www.nature.com/gim

\section{ACKNOWLEDGMENTS}

The authors thank all the patients and their families for their participation in our natural history protocol and donation of blood/ tissues for our studies; referring physicians, nurses and dietitians for their help with patient's evaluations; Isa Bernardini and Roxanne Fischer for processing patient samples; the nurses, research dietitians of the National Institutes of Health Clinical Research Center, and clinical fellows of the National Human Genome Research Institute genetics fellowship program for their help with patient care and dedication to clinical research. I.M, J.L.S., and
C.P.V were supported by the Intramural Research Program of the National Human Genome Research Institute, Bethesda, MD. J.M. was supported by the National Institutes of Health Clinical Center. The authors dedicate this manuscript to the memory of Harvey S. Mudd, who inspired us to study the role of methionine, methylation, and transsulfuration cycles in human physiology and disease. His stimulating discussions contributed significantly to our understanding of dietary effects in the management of cb/C-deficient patients.

\section{DISCLOSURE}

The authors declare no conflict of interest.

\section{REFERENCES}

1. Rosenblatt, DS, Fenton, WA. Inherited disorders of folate and cobalamin transport and metabolism. In: Scriver, CR, Sly, WS, Childs, B, et al. (eds). The Metabolic and Molecular Bases of Inherited Disease, 8th edn. Mc-Graw Hill: New York, 2001:3897-3933.

2. Levy HL, Mudd SH, Schulman JD, Dreyfus PM, Abeles RH. A derangement in B12 metabolism associated with homocystinemia, cystathioninemia hypomethioninemia and methylmalonic aciduria. Am J Med 1970;48:390-397.

3. Mudd SH, Levy HL, Abeles RH, Jennedy JP Jr. A derangement in B 12 metabolism leading to homocystinemia, cystathioninemia and methylmalonic aciduria. Biochem Biophys Res Commun 1969;35:121-126.

4. Carrillo-Carrasco N, Chandler RJ, Venditti CP. Combined methylmalonic acidemia and homocystinuria, cblC type. I. Clinical presentations, diagnosis and management. J Inherit Metab Dis 2012;35:91-102.

5. Carrillo-Carrasco N, Venditti CP. Combined methylmalonic acidemia and homocystinuria, cblC type. II. Complications, pathophysiology, and outcomes. J Inherit Metab Dis 2012;35:103-114.

6. Weisfeld-Adams JD, Bender HA, Miley-Åkerstedt A, et al. Neurologic and neurodevelopmental phenotypes in young children with early-treated combined methylmalonic acidemia and homocystinuria, cobalamin C type. Mol Genet Metab 2013;110:241-247.

7. Fischer S, Huemer M, Baumgartner M, et al. Clinical presentation and outcome in a series of 88 patients with the cblC defect. J Inherit Metab Dis 2014;37: 831-840.

8. Gizicki R, Robert MC, Gómez-López L, et al. Long-term visual outcome of methylmalonic aciduria and homocystinuria, cobalamin C type. Ophthalmology 2014:121:381-386.

9. Smith SE, Kinney HC, Swoboda KJ, Levy HL. Subacute combined degeneration of the spinal cord in $\mathrm{cblC}$ disorder despite treatment with B12. Mol Genet Metab 2006;88:138-145

10. Mc Guire PJ, Parikh A, Diaz GA. Profiling of oxidative stress in patients with inborn errors of metabolism. Mol Genet Metab 2009:98:173-180.

11. Pastore A, Martinelli D, Piemonte F, et al. Glutathione metabolism in cobalamin deficiency type C (cblC). J Inherit Metab Dis 2014;37:125-129.

12. Moreno-Garcia MA, Pupavac M, Rosenblatt DS, Tremblay ML, JeromeMajewska LA. The Mmachc gene is required for pre-implantation embryogenesis in the mouse. Mol Genet Metab 2014;112:198-204.

13. Tanpaiboon P, Sloan JL, Callahan PF, et al. Noncompaction of the ventricular myocardium and hydrops fetalis in cobalamin C disease. JIMD Rep 2013;10: 33-38.

14. Profitlich LE, Kirmse B, Wasserstein MP, Diaz GA, Srivastava S. High prevalence of structural heart disease in children with cblC-type methylmalonic aciduria and homocystinuria. Mol Genet Metab 2009;98:344-348.

15. Cerone R, Schiaffino MC, Caruso U, Lupino S, Gatti R. Minor facial anomalies in combined methylmalonic aciduria and homocystinuria due to a defect in cobalamin metabolism. J Inherit Metab Dis 1999:22:247-250.

16. Andersson HC, Marble M, Shapira E. Long-term outcome in treated combined methylmalonic acidemia and homocystinemia. Genet Med 1999;1:146-150.

17. Weisfeld-Adams JD, Morrissey MA, Kirmse BM, et al. Newborn screening and early biochemical follow-up in combined methylmalonic aciduria and homocystinuria, cblC type, and utility of methionine as a secondary screening analyte. Mol Genet Metab 2010;99:116-123.

18. Martinelli D, Dotta A, Massella L, et al. Cobalamin C defect presenting as severe neonatal hyperammonemia. Eur J Pediatr 2011;170:887-890. 


\section{ORIGINAL RESEARCH ARTICLE}

19. Harding CO, Pillers DA, Steiner RD, et al. Potential for misdiagnosis due to lack of metabolic derangement in combined methylmalonic aciduria/ hyperhomocysteinemia (cblC) in the neonate. J Perinatol 2003;23:384-386.

20. Mudd SH, Brosnan JT, Brosnan ME, et al. Methyl balance and transmethylation fluxes in humans. Am J Clin Nutr 2007;85:19-25.

21. Strauss KA, Morton DH, Puffenberger EG, et al. Prevention of brain disease from severe 5,10-methylenetetrahydrofolate reductase deficiency. Mol Genet Metab 2007:91:165-175.

22. Bodamer OA, Sahoo T, Beaudet AL, et al. Creatine metabolism in combined methylmalonic aciduria and homocystinuria. Ann Neurol 2005;57:557-560.

23. Schiff M, Benoist JF, Tilea B, Royer N, Giraudier S, Ogier de Baulny H. Isolated remethylation disorders: do our treatments benefit patients? I Inherit Metab Dis 2011;34:137-145.

24. Surtees R, Leonard J, Austin S. Association of demyelination with deficiency of cerebrospinal-fluid S-adenosylmethionine in inborn errors of methyl-transfer pathway. Lancet 1991;338:1550-1554.

25. Lerner-Ellis JP, Anastasio N, Liu J, et al. Spectrum of mutations in MMACHC, allelic expression, and evidence for genotype-phenotype correlations. Hum Mutat 2009;30:1072-1081.

26. Schakel, SF Maintaining a nutrient database in a changing marketplace: keeping pace with changing food products-a research perspective. J Food Compost Anal 2001;14:315-322.

27. National Research Council. Dietary Reference Intakes for Energy, Carbohydrate, Fiber, Fat, Fatty Acids, Cholesterol, Protein, and Amino Acids (Macronutrients). Washington, DC: National Academies Press, 2005.

28. Strauss KA, Wardley $B$, Robinson $D$, et al. Classical maple syrup urine disease and brain development: principles of management and formula design. Mol Genet Metab 2010;99:333-345.

29. Strauss KA, Brumbaugh J, Duffy A, et al. Safety, efficacy and physiological actions of a lysine-free, arginine-rich formula to treat glutaryl-CoA dehydrogenase deficiency: focus on cerebral amino acid influx. Mol Genet Metab 2011;104:93-106.

30. Smith QR, Stoll JS. Blood-brain barrier amino acid transport. In: Pardridge WM (ed). Introduction to the Blood-Brain Barrier. Cambridge University Press: Cambridge, UK, 1998:188-197.

31. Enns GM, Barkovich AJ, Rosenblatt DS, et al. Progressive neurological deterioration and $\mathrm{MRI}$ changes in cblC methylmalonic acidaemia treated with hydroxocobalamin. J Inherit Metab Dis 1999;22:599-607.

32. Rossi A, Cerone R, Biancheri R, et al. Early-onset combined methylmalonic aciduria and homocystinuria: neuroradiologic findings. AJNR Am J Neuroradiol 2001;22:554-563.
33. Scott JM, Dinn JJ, Wilson P, Weir DG. Pathogenesis of subacute combined degeneration: a result of methyl group deficiency. Lancet 1981;2: 334-337.

34. Tsina EK, Marsden DL, Hansen RM, Fulton AB. Maculopathy and retinal degeneration in cobalamin C methylmalonic aciduria and homocystinuria. Arch Ophthalmol 2005;123:1143-1146.

35. Debray FG, Boulanger $Y$, Khiat A, et al. Reduced brain choline in homocystinuria due to remethylation defects. Neurology 2008;71:44-49.

36. Rubin RA, Ordonez LA, Wurtman RJ. Physiological dependence of brain methionine and S-adenosylmethionine concentrations on serum amino acid pattern. J Neurochem 1974;23:227-231.

37. Harper AE, Miller RH, Block KP. Branched-chain amino acid metabolism. Annu Rev Nutr 1984;4:409-454.

38. Manoli I, Venditti, C. Branched chain amino acid disorders. In: Lee B, Scaglia F (eds). Inborn Errors of Metabolism. From Neonatal Screening to Metabolic Pathways. Oxford University Press: NY, 2015.

39. Zinnanti WJ, Lazovic J, Housman C, et al. Mechanism of age-dependent susceptibility and novel treatment strategy in glutaric acidemia type I. J Clin Invest 2007;117:3258-3270.

40. Sauer SW, Opp S, Hoffmann GF, Koeller DM, Okun JG, Kölker S. Therapeutic modulation of cerebral L-lysine metabolism in a mouse model for glutaric aciduria type I. Brain 2011;134(Pt 1):157-170.

41. Bröer S. The SLC6 orphans are forming a family of amino acid transporters. Neurochem Int 2006:48:559-567.

42. Sloan JL, Mager $\mathrm{S}$. Cloning and functional expression of a human $\mathrm{Na}(+)$ and $\mathrm{Cl}(-)-$ dependent neutral and cationic amino acid transporter $\mathrm{B}(0+)$. J Biol Chem 1999;274:23740-23745

43. Michalec $K$, Mysiorek $C$, Kuntz $M$, et al. Protein kinase $C$ restricts transport of carnitine by amino acid transporter $\operatorname{ATB}(0,+)$ apically localized in the blood-brain barrier. Arch Biochem Biophys 2014;554:28-35.

44. Acosta PB, Yannicelli S, Singh R, et al. Intake and blood levels of fatty acids in treated patients with phenylketonuria. J Pediatr Gastroenterol Nutr 2001;33:253-259.

45. Gil-Campos M, Sanjurjo Crespo P. Omega 3 fatty acids and inborn errors of metabolism. Br J Nutr 2012;107(suppl 2):S129-S136

46. Riediger ND, Othman RA, Suh M, Moghadasian MH. A systemic review of the roles of n-3 fatty acids in health and disease. J Am Diet Assoc 2009;109: 668-679.

47. Din JN, Harding SA, Valerio CJ, et al. Dietary intervention with oil rich fish reduces platelet-monocyte aggregation in man. Atherosclerosis 2008;197: 290-296. 